\title{
The Structural Relation between the Topological Manifold I: Connectedness
}

\author{
Shri H.G .Haloli \\ Research Student, Karnataka University, Dharwad,Karnataka, India
}

\begin{abstract}
We conduct a study on Topological Manifold and some of its properties Theorems, structures on topological manifold and its structural characteristics. The main properties in Topological Manifold is connectedness. This connectedness are studied the path connected, locally connected,locally path connected with cut point and punctured point and punctured space on Topological Manifold.
\end{abstract}

Key-Word: - Connectedness, cut point, locally connected, locally path connected, path-connected, punctured space.

\section{INTRODUCTION}

We are using basic concepts like, connectedness, subspace of Topological manifold, Topological space like(sub space) product space, quotient space, Equivalence Space .The Connectivity of Topological Manifold Plays an important role .In connectivity of Topological manifold William Basener[13] introduced the basic concepts of all types of connectivity inTopological Manifold. Also the concept of path connectivity is discussed by Lawrence Conlon [7].

Devender Kumar Kamboj and Vinod Kumar [3] [4] introduced the concept of cut points which plays a very important role in Topological space. To study cut point, a topological space is assumed to be connected. The idea of cut points in a topological space comes dates back to 1920's.After 2010 by Devender Kumar Kamboj and Vinod Kumar[3][4]uplifted the concepts of cut- points. This cut point concept plays an important role in my work.

The topological subspace is also important part of the work.

An injective continuous map that is a homeomorphism onto its image in the subspace topology is called topological embedding. If $f: \mathrm{A} \rightarrow \mathrm{X}$ is such a map we can think of the image set $\mathrm{f}(\mathrm{A})$ as a homeomorphic copy of $\mathrm{A}$ embedded in $\mathrm{X}$. The main role of path connectedness in a connected space gives the non- cut point connected so called strongly connected.

We are imbibing these following basic definitions in our discussion

1). Topological Manifolds

2). 2)Connectedness

3). Path connected spaces

4). Locally connected

5). Locally path connected

6). Of all the spaces which one studies in topology the Euclidean space and their subspace are the most important also the metric spaces $\mathrm{R}^{\mathrm{n}}$ serve as a topological model for Euclidean space $\mathrm{E}^{\mathrm{n}}$ for finite dimensional vector space.

In the section 2, we defined some basic concepts and proved some theorems.

In section 3, we proved main part of paper, this section we defined cut point, punctured points and connectedness property of Topological manifold $\mathrm{M}$.

\section{SOME BASIC DEFINITIONS}

Definition 2.1 [11][13][7]

A Topological space $\mathrm{X}$ is a Manifold of dimensions $\mathrm{n}$ (an- $\mathrm{n}$ - manifold) if

i) $\quad \mathrm{X}$ is locally Euclidean and $\operatorname{dim}$ of $\mathrm{X}=\mathrm{n}$

ii) $\quad X$ is $2^{\text {nd }}$ countable

Iii) $\quad \mathrm{X}$ is Hausdorff

Here Hausdorff means that any two distinct points lie in disjoint open sets but in the same space. Second countable means that there is a countable family of open subsets is the union of a subfamily. Locally homeomorphic or local Euclidean means that every point has an open neighborhood homeomorphic with an open subset of $\mathrm{R}^{\mathrm{n}}$ 


\section{Example 2.2}

Let $\mathrm{M}$ be an open subset of $\mathrm{R}^{\mathrm{n}}$ with the subspace topology then $\mathrm{M}$ is an $\mathrm{n}$-manifold

\section{Example 2.3}

The simplest examples of manifold not homeomorphic to open subsets of Euclidean space are the circle $S^{1}$ and 2-spheres $S^{2}$ which way be defined to be all points of $E^{2}$ or of $E^{3}$ respectively which are at unit distance from a fixed point $\mathrm{O}$.

\section{Definition 2.4connectedness [12] [13]}

A topological space $\mathrm{X}$ is said to be connected if the only subset of $\mathrm{X}$ that are both open and closed are itself and the empty set.

\section{Example 2.5}

$\mathrm{R}$ is connected and any interval in $\mathrm{R}$ is connected.

\section{Definition 2.5}

A subset $\mathrm{A}$ of a topological space $\mathrm{Y}$ is called a component of $\mathrm{y}$ if $\mathrm{A}$ is connected (in the sub-space topology) and if there is no connected subset of $\mathrm{y}$ that properly contains A.

i.e. A topological space is connected if it has only one piece. The connected pieces are called components.

\section{Definition2.6 [12] [8]}

A Topological Space $\mathrm{X}$ is said to disconnected if there exists two non-empty subset A, B of X satisfying

(ii) $\mathrm{A} \cap \mathrm{B}=\varnothing$

$$
\text { (i) } \mathrm{A} U B=\mathrm{X}
$$

(iii) $\mathrm{A}$ and $\mathrm{B}$ are both open and closed

\section{Definition2.7.Path Connected [8] [12][13]}

A Topological Space $X$ is path connected if given any two -points $x, y \in X$, there exists a map $\gamma:[0,1] \rightarrow \quad X$ such that $\gamma(0)=\mathrm{x}$ and $\gamma(1)=\mathrm{y}$. The map $\gamma$ is called path in $\mathrm{X}$ from $\mathrm{x}$ toy

Particularly a surface is a path connected if given any two points $x, y \in S$ there is a map $\gamma[0,1] \rightarrow S$ such that $\gamma(0)=\mathrm{x}$ and $\gamma(1)=\mathrm{y}$. The map $\gamma$ is called a path in $\mathrm{S}$ from $\mathrm{x}$ toy.

\section{Example 2.8}

(i)Fish-net (ii) Networked transport on the globe

There exists at least one path from one goods to any other goods directly or indirectly

\section{Example 2.9}

The standard example of a topological set that is connected but not path connected.

Let $\mathrm{S}$ be the set.

$\mathrm{Y}=\operatorname{Sin}(1 / \mathrm{x})$

The following figure shows a space that is locally connected but not locally path connected

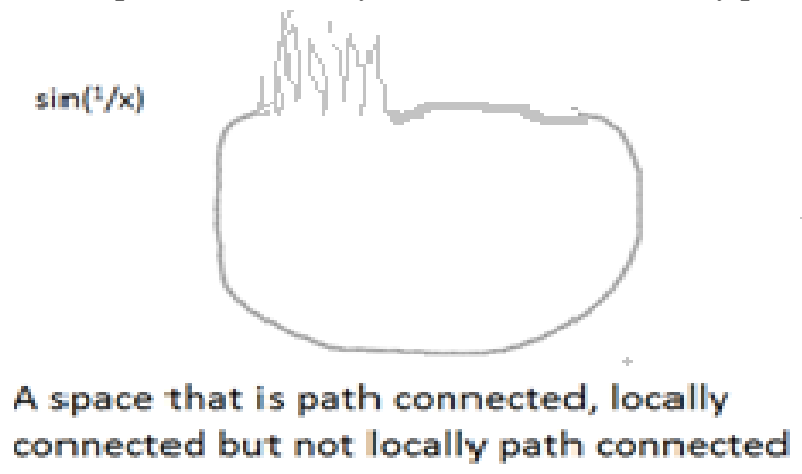

Fig:2.1

$S=\left\{\left(x, \sin ^{1} / x\right) \in R^{2} / x \in R\right\} \cup\left\{(0, t) \in R^{2} / t \in[-1,1]\right\}$

$\mathrm{X}$ is a point in a topological space $\mathrm{X}$ 


\section{Definition 2.10 [13]}

A Topological space $\mathrm{X}$ is locally connected if every $\mathrm{x} \epsilon \mathrm{X}$ has a connected neighborhood. A Space $\mathrm{X}$ is locally path connected if every $\mathrm{x} \epsilon \mathrm{X}$ has a path connected neighborhood.

\section{Example 2.11}

Any open subset $\mathrm{O} \subseteq \mathrm{R}^{\mathrm{n}} \quad$ is locally path connected for each $\mathrm{x} \epsilon$ O. There is an open ball $\mathrm{B}_{\mathrm{r}}(\mathrm{x}) \subseteq \mathrm{O}$ and this ball is a path connected neighborhood of $\mathrm{x}$.

The space $\{\mathrm{O}\} \cup[1 / \mathrm{n} \in \mathrm{R} / \mathrm{n} \in \mathrm{N}]$ is not locally connected or locally path connected.

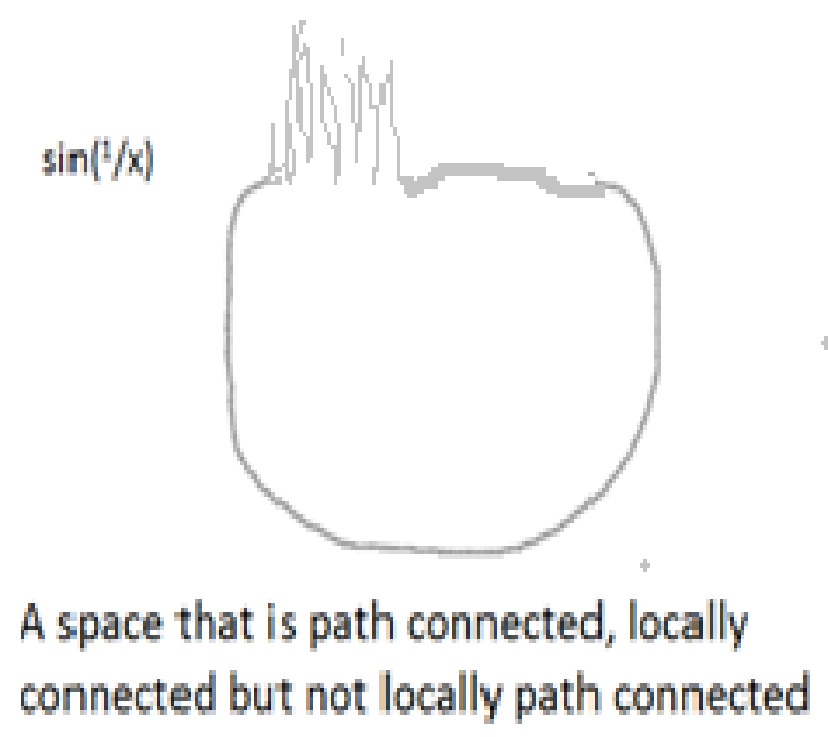

Fig:2.2

This space is obtained by connecting the tail of the graph of $\sin (1 / x)$ to the origin .Any neighborhood of the origin is not path connected and hence the space is not locally path connected.

Theorem 2.12 [7] [John Lee]

Every manifold is locally path connected

Theorem 2.13 [12]

Let $\mathrm{X}$ be an open subset of $\mathrm{R}^{\mathrm{n}}$.If $\mathrm{X}$ is connected then $\mathrm{X}$ is path connected

\section{Theorem 2.14}

Every connected space need not be locally connected

\section{Proof}

Let $\mathrm{X}$ be a connected space,

\section{Case I}

Suppose, $\mathrm{X}$ is an open subset of $\mathrm{R}^{\mathrm{n}}$, then by Theorem 2.13,

$\mathrm{X}$ is path connected .By above example 2.11 ,every path connected space is locally connected

\section{Case II}

Suppose $\mathrm{X}$ is not an open subset of $\mathrm{R}^{\mathrm{n}}$ then $\mathrm{X}$ is not locally connected because there does not exists connected neighborhood of $\mathrm{X}$.

Therefore, for all $\mathrm{y} \neq \mathrm{x}$, and $\mathrm{x}, \mathrm{y} \in \mathrm{X}, \mathrm{N}_{\mathrm{y}}(\mathrm{X}) \& \mathrm{~N}_{\mathrm{x}}(\mathrm{X})$ are neighborhood of $\mathrm{y}$ and $\mathrm{x}$ respectively such that $\mathrm{N}_{\mathrm{y}}(\mathrm{X})$ $\cap \mathrm{N}_{\mathrm{x}}(\mathrm{X})=\varnothing$ which are disjoint neighborhood. Hence forming a disconnected neighborhood. This implies $\mathrm{X}$ is not locally connected.

\section{Theorem $2.15[13]$}

Every path connected space is connected converse need not be true. 


\section{Theorem 2.16}

Every locally path connected space is locally connected (converse not true)

\section{Proof}

Let $\mathrm{X}$ be a locally path connected space.

To prove that, $\mathrm{X}$ is locally connected

As $X$ is locally path connected then there exists a path $\gamma\left[\begin{array}{ll}0 & 1]\end{array} \rightarrow\right.$ such that $\gamma(0)=X, \gamma(1)=1$. This shows that there exists a path $\gamma$ from $\mathrm{x}$ to $\mathrm{y}$ in $\mathrm{X}$.

Let $\mathrm{N}_{\mathrm{x}}(\mathrm{X})$ \& $\mathrm{N}_{\mathrm{y}}(\mathrm{X})$ be two local neighborhood of $\mathrm{x}$ and $\mathrm{y}$ respectively. As $\mathrm{X}$ is path connected space.

Then there exists a path from $\mathrm{N}_{\mathrm{x}}(\mathrm{X})$ to $\mathrm{N}_{\mathrm{y}}(X) \cdot \mathrm{N}_{\mathrm{x}}(\mathrm{X})$ and $\mathrm{N}_{\mathrm{y}}(\mathrm{X})$ are connected by path this implies $\mathrm{X}$ is locally connected

\section{Example 2.17}

$\mathrm{Y}=\sin (1 / \mathrm{x}) \operatorname{see}[13] \mathrm{p}-66)$

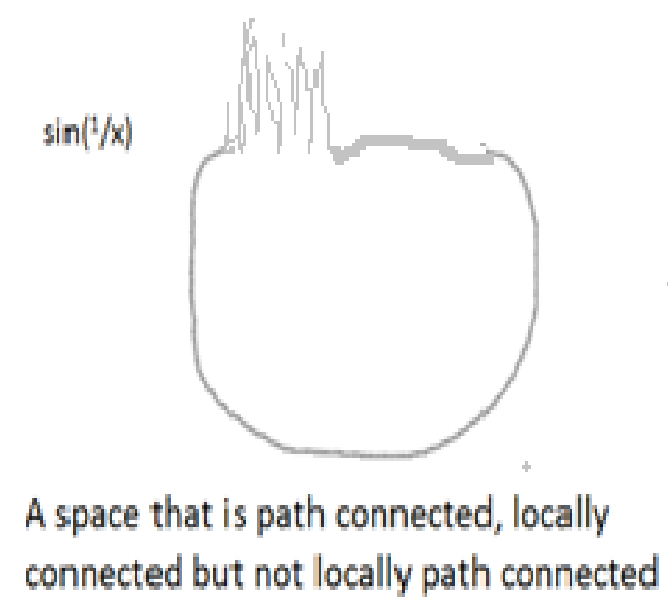

Fig:2.3

\section{Theorem 2.18}

The product of two locally connected space is locally connected.

\section{Proof}

Let $\mathrm{X}$ and $\mathrm{Y}$ be two locally connected spaces.

$\mathrm{N}_{\mathrm{x}}(\mathrm{X})$ and $\mathrm{N}_{\mathrm{y}}(\mathrm{X})$ are two neighborhoods of $\mathrm{x}$ and $\mathrm{y}$ respectively.

The production of two locally space is $\mathrm{X} \mathrm{X} Y$.

This space $\mathrm{X} x \mathrm{Y}$ has connected local neighborhood. That is each point $(\mathrm{x}, \mathrm{y}) \epsilon(\mathrm{X} \mathrm{x} \mathrm{Y})$ has a connected neighborhood. Which contains point $(x, y)$ say that is $O x y . N_{x}(X) \cap N_{y}(X) \neq \varnothing$

Oxy is connected open subset of $\mathrm{R} x \mathrm{R}$ which is connected. There exists a local neighborhood of $\mathrm{Oxy} \epsilon \mathrm{X} x \mathrm{Y}$. This shows that, $\mathrm{X} \times \mathrm{Y}$ is locally connected. Hence the product of two locally connected space is locally connected.

\section{Theorem 2.19}

The product of two path connected space is path connected.

\section{Proof}

Let $\mathrm{X}$ and $\mathrm{Y}$ are two path connected spaces. There exists a path $\gamma$ and $\sigma$ in $\mathrm{X}$ and $\mathrm{Y}$ such that $\gamma$ isa path between any two points say $\mathrm{x}_{1}, \mathrm{x}_{2}$ in $\mathrm{X} \sigma$ is path between any two points in $\mathrm{Y}$ say $\mathrm{y}_{1}, \mathrm{y}_{2}$. As the product spaces $(\mathrm{x}, \mathrm{y}) \epsilon \mathrm{X}$ $\mathrm{x} Y$ for all $\mathrm{x}_{1}, \mathrm{x}_{2} \in \mathrm{X}, \mathrm{y}_{1}, \mathrm{y}_{2} \in \mathrm{Y}$ if there exists a continuous map $\mathrm{P}(\mathrm{x}, \mathrm{y})=\mathrm{x}$ or $\mathrm{y}$

i.e. $P_{i}\left(x_{1} \times x_{2} \times x_{3} \ldots x_{n}\right)=x_{i}$ for $i$

By the definition of product of path.

If $\gamma$ and $\sigma$ are paths in X such that the end point of $\gamma$ is the beginning point of $\sigma$, then we combine $\gamma$ and $\sigma$ to form a new path that fallows $\sigma$ and then $\gamma$ 


$$
\begin{array}{llll}
\gamma \circ \sigma & \{\sigma(2 \mathrm{~S}) & \text { if } & 0 \leq \mathrm{S} \leq 1 / 2 \\
=\{\gamma(2 \mathrm{~S}-1) & \text { if } & 1 / 2<\mathrm{S} \leq 1
\end{array}
$$

The path $\gamma \circ \sigma$ is called the product of $\gamma$ and $\sigma$

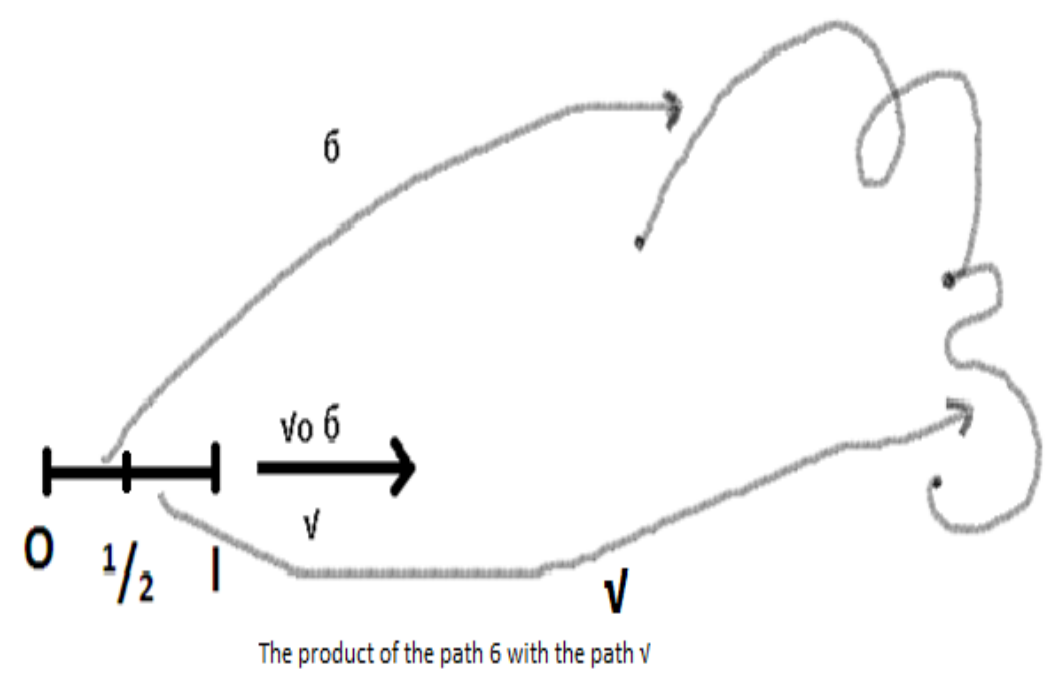

Fig:2.3

This shows that the product of two path connected space is path connected space is the path connected.

i.e. There exists any path from one point to any point in $X \times Y, P(0)=x_{1} x_{y_{1}}$ and $P(I)=x_{2} x_{2} y_{2}$ for all $x_{i} \epsilon X, y_{i} \epsilon$ $\mathrm{Y}$ in between $\mathrm{P}[0,1] \rightarrow \mathrm{X} \times \mathrm{Y}$.

\section{CUT POINT}

The concept of cut point plays a very important role in topological space X. In this paper we study the same cut point concept in connected topological spaces.

Cut points in some connected topological space have been studied by Honari and Bahrampour[2] and Devender Kumar Kamboj [4] H. G. Haloli[6].

If $x \in \in X$ is such that $[x]$ is closed. We say that $x$ is a closed point of X. D. Kumar and V. Kumar[3] introduced. A connected space having only finitely many closed point is proved have at least two non-cut points and such non-indiscrete space which has exactly two non-cut points is proved homeomorphic to a finite subspace of the Khalimsky line.

\section{Definition 3.1}

A point $\mathrm{x}$ in a topological space is said to cut point if by removal of $\mathrm{x}, \mathrm{X}$ becomes disconnected as separation

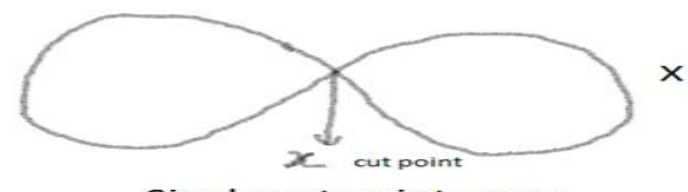

Single cut point space

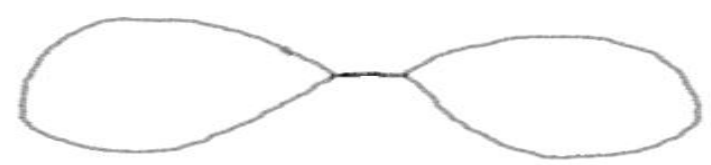

A space with more than one (same) cut point space

Fig: 3.1, 3.2

\section{Definition 3.2 Strong cut point:}

A point $\mathrm{X}$ of a space $\mathrm{X}$ is called a strong cut point if the removal of a cut point from space $\mathrm{X}, \mathrm{X}$ becomes separated sets, which are connected. 
That is a point $\mathrm{x}$ of a topological space $\mathrm{X}$ is said to be a strong cut point if

(i) $\quad \mathrm{X}-\{\mathrm{x}\}$, is disconnected

(ii) (ii) $A_{x} \cap B_{x}=x$

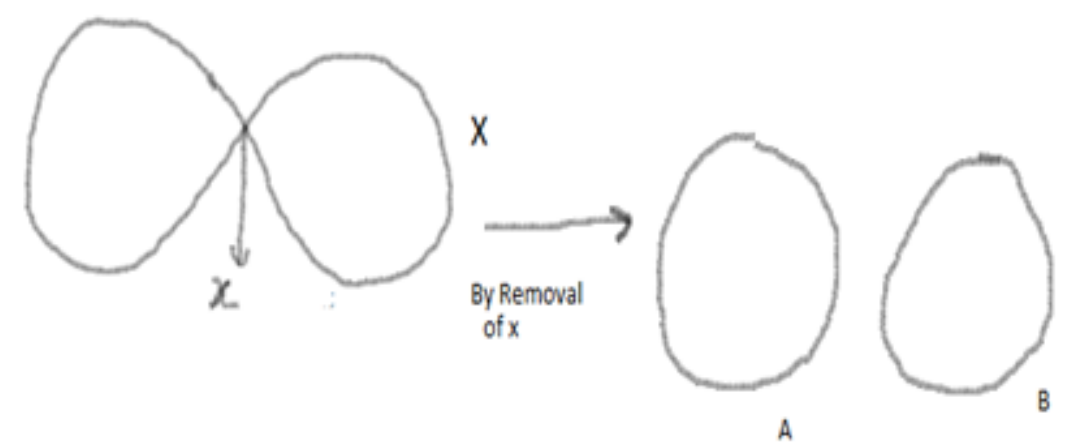

Both are connected

Fig:3.3 i.e. A connected space contains single cut point such point is called strong cut point.

Definition 3 .3. Punctured point:

A point $x$ in topological space or surface $X$ or $S$ is called punctured point if $X-\{x\}$ or $S-\{x\}$ is connected.

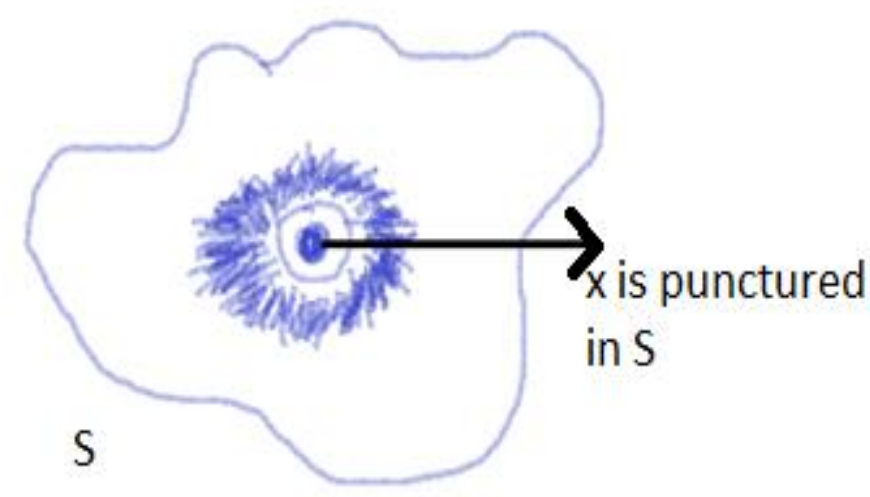

plane surface (hole)

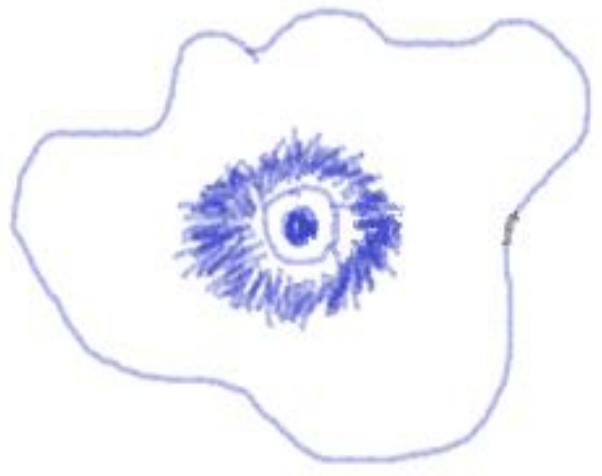

Any surface (punctured point)

Fig: 3.4 Definition 3.4 Punctured Neighborhood

A punctured neighborhood of a point $\mathrm{x}$ is a neighborhood of $\mathrm{x}$ minus $\{\mathrm{x}\}$

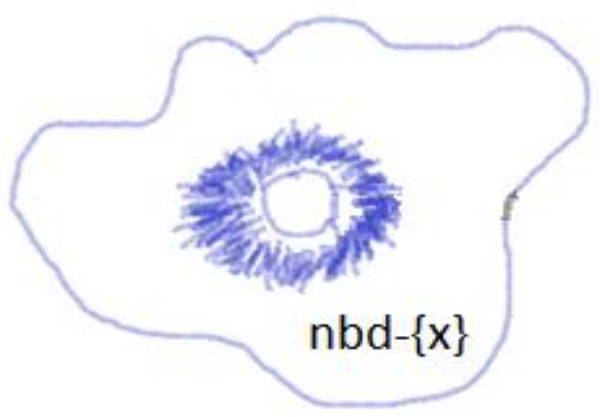

Fig: 3.5 


\section{Example 3.5}

For instant , the interval $(-1,1)=\{\mathrm{y}:-1<\mathrm{y}<1\}$ is a neighborhood of $\mathrm{x}=0$ in the real line, So the set $(-1,0) \mathrm{U}(0,1)=(-$ $1,1)-\{0\}$ is a punctured neighborhood of $\mathrm{O}$.

\section{Remarks 3.6}

1) The Set of all neighborhood of punctured point are non-cut points of $X$.

2) The Set of all the neighborhood of $x \in X$ are removed or deleted then the space becomes a non- convex space but $\mathrm{X}$ is connected.
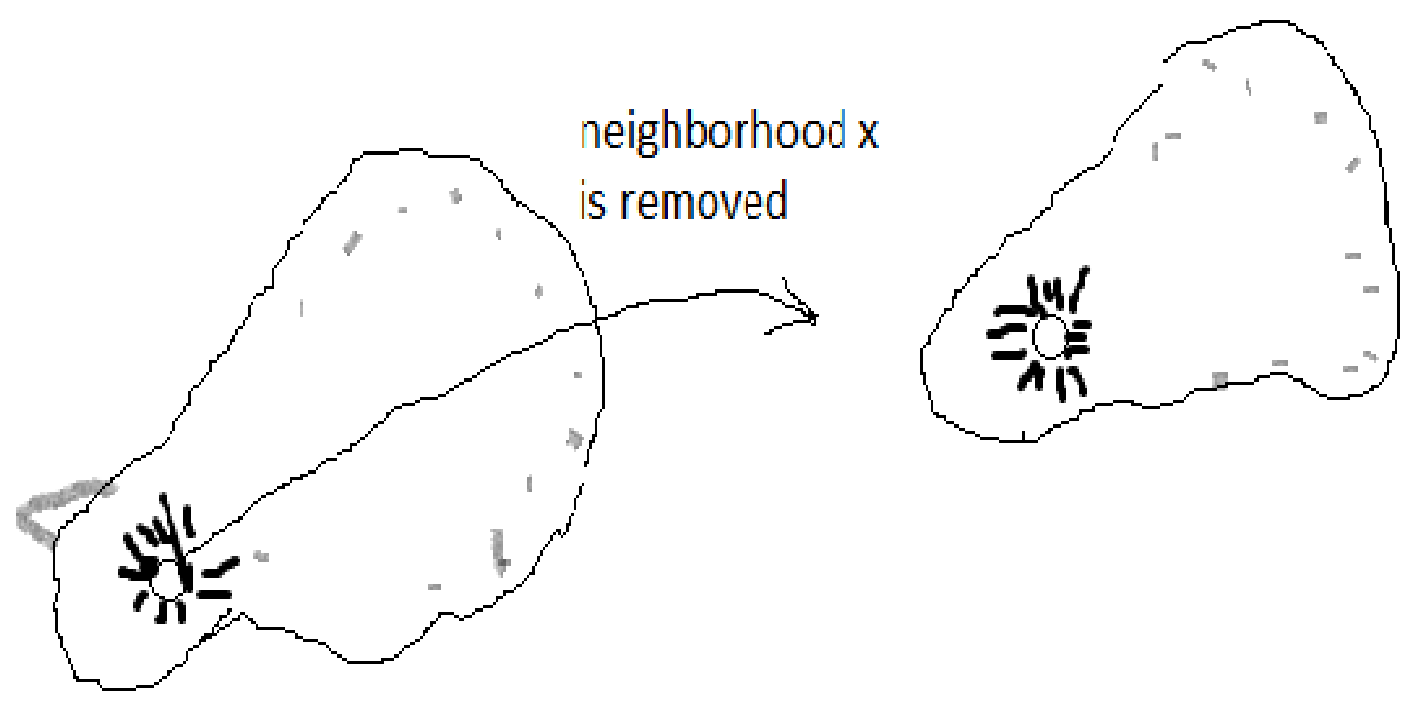

Fig:3.6

\section{Theorem 3.7}

Let $\mathrm{X}$ be a non -cut point space, if $\mathrm{X}$ is connected then $\mathrm{X}$ is path connected.

\section{Proof}

Here $\mathrm{X}$ is non- cut point space. This implies, $\mathrm{X}$ connected. A space connected without any cut point, mean $\mathrm{X}$ is strong connected[see definition 3.8] Thenthere exists a path from any point $\mathrm{x}$ of $\mathrm{X}$ to another point $\mathrm{y} \epsilon \mathrm{X}$. This shows $\mathrm{X}$ path is connected.

As $X$ is connected with non- cut points is open in $\mathrm{R}^{\mathrm{n}}$.

Choose any two points $\mathrm{x}, \mathrm{y} \epsilon \mathrm{X}$ which are non cut points of $\mathrm{X}$ then there exists a path between them. This implies $\mathrm{X}$ is path connected.

\section{Definition 3.8 Strongly connected space}

A connected space $\mathrm{X}$ is said to be strongly connected if

i. There does not exist a cut point

ii. It may be an existing punctured point

iii. There exists a path between any two points in $\mathrm{X}$

A connected space $\mathrm{X}$ is said to be strongly connected if there is a path between any two points in $\mathrm{X}$ and there does not exists finite cut points.

\section{Lemma 3.9}

Every strongly connected space is connected, converse need not be true.

\section{Proof}

Let $\mathrm{X}$ be a strongly connected space.

By definition of strongly connected space, there does not exist a cut point in $\mathrm{X}$

Therefore, let $\mathrm{x}$ be punctured point in $\mathrm{X}, \mathrm{X}-\{\mathrm{x}\}$ is a connected space by definition of punctured point.

As $\mathrm{X}$ is strongly connected there exists a path between any two points in $\mathrm{X}$.

A strongly connected space does not exists separation which shows $\mathrm{X}$ is connected.

By Theorem 2.15, every path connected space is connected. 


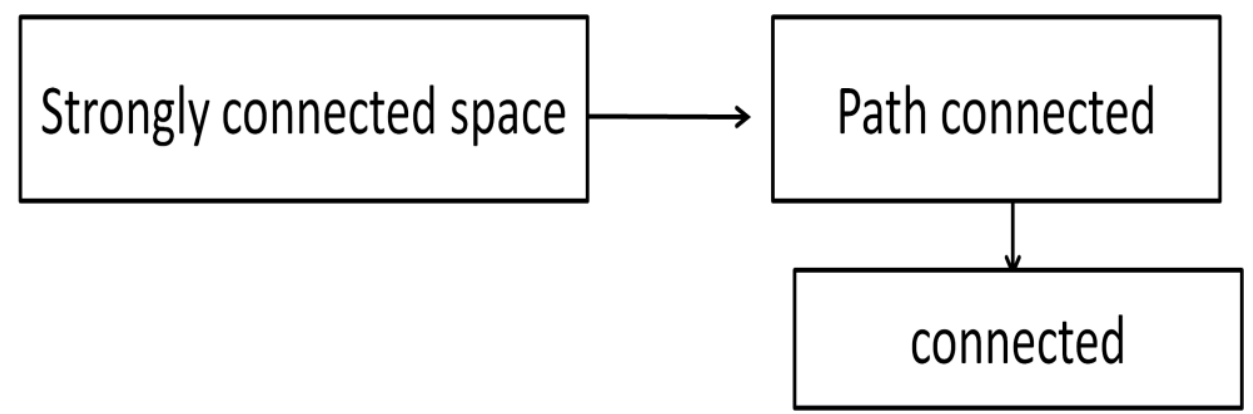

Fig:3.7

Converse of Theorem 2.15 need not be true.

i.e. connectedness does not implies path connectivity ,path connected space fail for $\mathrm{X}$. Then $\mathrm{X}$ is not strongly connected

\section{Lemma 3.10}

Every strongly connected space is path connected converse is true for only path connected space with non- cut points.

\section{Proof}

Let $\mathrm{X}$ be strongly connected space, By Theorem 2.15, X is path connected. As X is path connected there exists a path between any two points in $\mathrm{X}$.

But,as $\mathrm{X}$ is connected if $\mathrm{X}$ does not contain cut points then $\mathrm{X}$ is path connected.

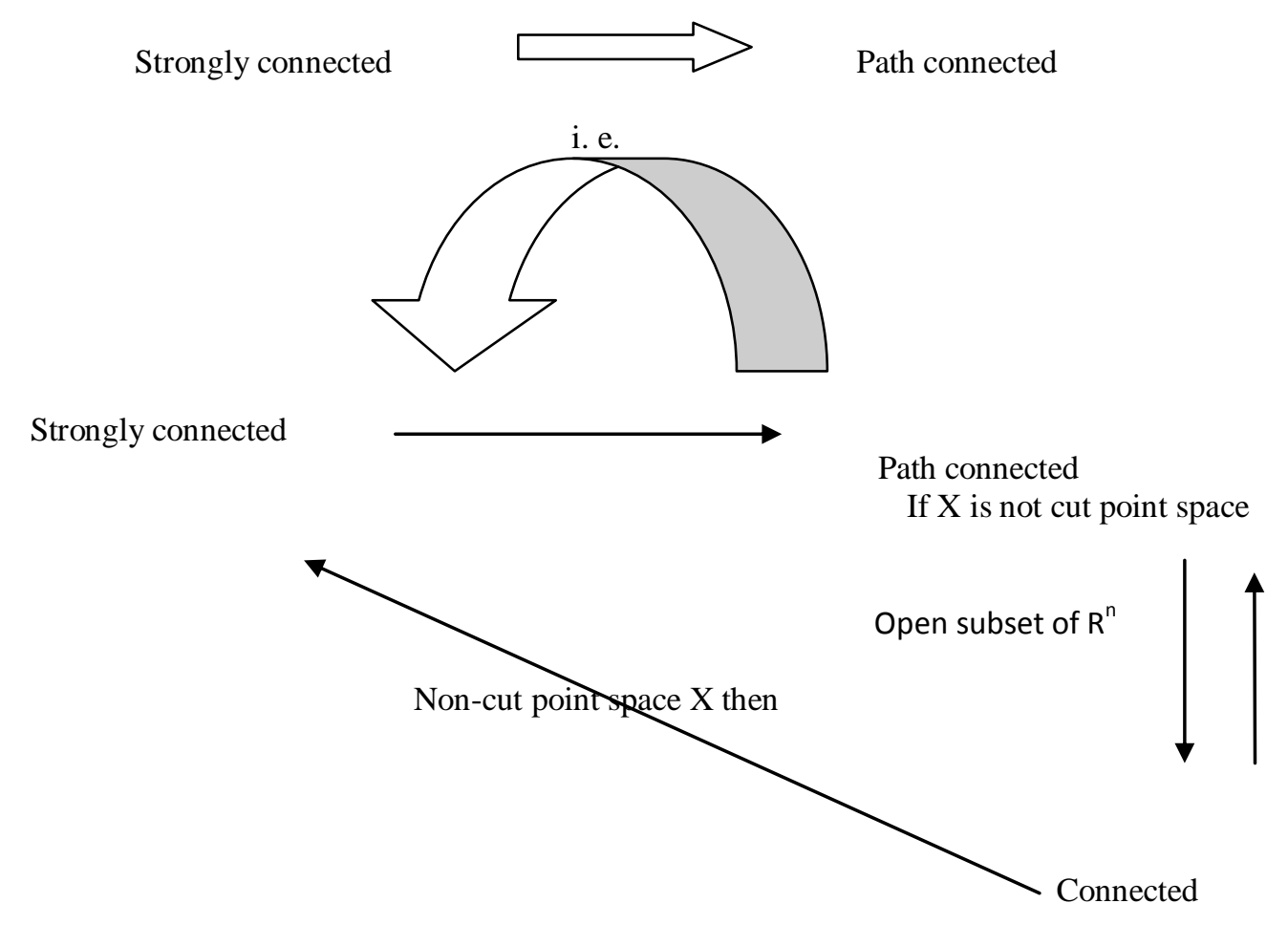

Fig 3.8

\subsection{Notations}

We shall mean a topological space $\mathrm{X}$ contains at least two points.

Let $\mathrm{X}$ be topological space which is connected, $\mathrm{x}$ be a cut point of $\mathrm{X}$, where $\mathrm{x}$ is closed.

Then $\mathrm{Ct} X$ - denote the set of all cut points of a space $\mathrm{X}, \mathrm{A} / \mathrm{B}$ from a separation of a space $\mathrm{X}$ by cut point $\mathrm{X}$. We say that each one of $A$ and $B$ is separating set of $X$, A separation $A / B$ of $X-\{x\}$ is denoted by $A x / B x$ if the dependence of the separation on $\mathrm{x}$ is to be specified. 
$A_{x} *$ is issued for the set $A x u\{x\}$ similarly for a connected subset $Y$ of $X-\{x\}, A x(Y)$ is used to denote the separating subset of $\mathrm{X}-\{\mathrm{x}\}$ containing $\mathrm{Y}$.

A connected space with $X=$ ct $X$ is called a cut point space.

Let $\mathrm{a}, \mathrm{b} \in \mathrm{X}$, a point $\mathrm{x} \in \operatorname{ctX}-\{\mathrm{a}, \mathrm{b}\}$ is said to be a separating point between $\mathrm{a}$ and $\mathrm{b}$ if for same separation $\mathrm{Ax} / \mathrm{Bx}$ of $X-\{x\}$

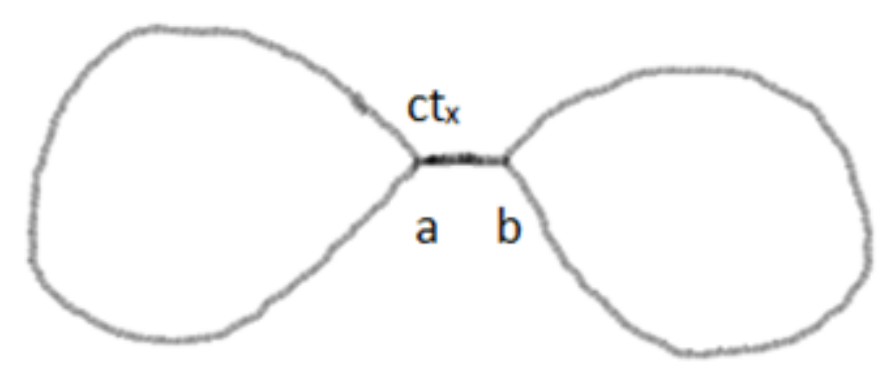

Fig: 3.9

- $\quad \mathrm{S}(\mathrm{a}, \mathrm{b})$ is used to denote the set of all separating points between $\mathrm{a}$ and $\mathrm{b}$

- $\quad \mathrm{S} \mathbf{I}$ a , b I Adjoin the point $\mathrm{a}$ and $\mathrm{b}$ to $\mathrm{S}(\mathrm{a}, \mathrm{b})$

A space $X$ is called a space with end points if there exists $a$ and $b$ in $X$ such that $X=s|a, b|$

\section{Lemma 3.12 [3]}

Let $\mathrm{X}$ be a connected space and $\mathrm{a}, \mathrm{b}, \mathrm{x}, \mathrm{y}, \in \mathrm{X}$

1) If $t \in s(x, y)-s|a, b|$ and $X-\{t\}=A_{t}(x) \subseteq B_{t}(Y)$ then

a) If $a \in A_{t}(x)$ then $b \in B_{t}(Y)$

b) Either $\{a, b\} C A_{t}(x)$ or $\{a, b\} c B_{t}(y)$

2) If $x, y \in S|a, b|$ then $S|x, y| \subseteq S|a, b|$

\section{Theorem 3.13[3]}

Let $\mathrm{X}$ is a connected space with end points then $\mathrm{X}$ has exactly two non cut points and every cut point of $\mathrm{X}$ is strong cut point.

\section{Now we redefine the path by using a cut point.}

The pathin a connected space, is the set of all cut points in $\mathrm{X}$ such that each preceding points is adjoin with next point which forms a continuous limits points of space.

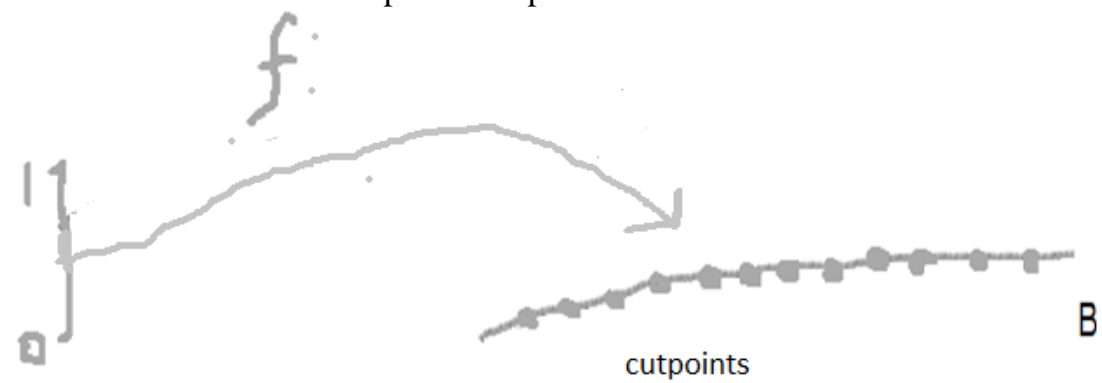

A

Curves in $\mathrm{R}^{\mathrm{n}}$ which is path with continous cutpoint

Fig:3.10

Curves in $\mathrm{R}^{2}$ which is path with continuous cut points.

\section{Lemma 3.14}

Let $\mathrm{X}$ be a topological space which is connected and $\mathrm{x}, \mathrm{y} \in \mathrm{X}$, then

1) If $x, y$ are the non- cut points of $X$ then there exacts a path between $x$ and $y$

2) If $x \in X$, a set of cut points of $X$ (from $x$ to $y$ ) then there exists at least one path which covers cut points 


\section{Proof}

Let $\mathrm{X}$ be a topological space which is connected by lemma 3.12 shows that there exits exactly two non -cut points of X. By definition, thereexists a path between $\mathrm{x}$ and $\mathrm{y}$. The ct $\mathrm{x}$ forms an open sub set of $\mathrm{X}$ which is connected then there is a path connected space. Therefore any two points $\mathrm{x}$ and $\mathrm{y}$ of $\mathrm{X}$ which are non -cut points.Therefore, $\mathrm{X}$ exists a path between them. Any point except cut point are adjoin with at least two neighborhood points which are open in X.Also in path, this implies each path is connected, which forms a dense in $\mathrm{X}$. Which implies, $\mathrm{X}$ is closed

$\Rightarrow \quad \mathrm{X}$ is closed

$\Rightarrow \mathrm{X}$ is connected

D. Kumar Kumboj and V. Kumar [3] [ Theorem 3.1] introduced, There exists path between two end points in X, which are non- cut points. That path passing through all cut points in X.

\section{Theorem: 3.15}

Let $\mathrm{X}$ is a strongly connected space with end points them there exists a path between them.

\section{Proof}

As $\mathrm{X}$ be a strongly connected space which contains $\mathrm{x}$, $\mathrm{y}$ which are end points we have by lemma 3.9 every strongly connected space is connected and by Definitions of strongly connected space show that there exista path between $\mathrm{x}$ and $\mathrm{y}$ in $\mathrm{X}$.

\section{Definition 3.16 weakly connected spaces}

A topological space $\mathrm{X}$ is said to be weekly connected if $\mathrm{X}$ is not strongly connected.

Weekly connected topology is locally defined but strongly connected property is globally.

\section{Theorem 3.17}

Let $\mathrm{X}$ be a connected space than following are equivalent

i) $\quad \mathrm{X}$ has exactly two non -cut points

ii) There exists a path between two non- cut points in $\mathrm{X}$

iii) If $\mathrm{X}$ is weakly connected than each path contains at least are cut points.

\section{Proof:}

Assume that (i) hold

I.e. Assume, $\mathrm{X}$ has exactly two non- cut points.Let $\mathrm{X}$ be a connected space. Then by Definition of connected space and path space and Theorem 3.15 and lemma 3.14 in proves (i) $\rightarrow$ (ii)

Assume that, there exists a path between any two points in X and $\mathrm{X}$ is weakly connected then there exists some cut points in space $\mathrm{X}$ but space is connected also path connected than exactly each path is passing through at least one point

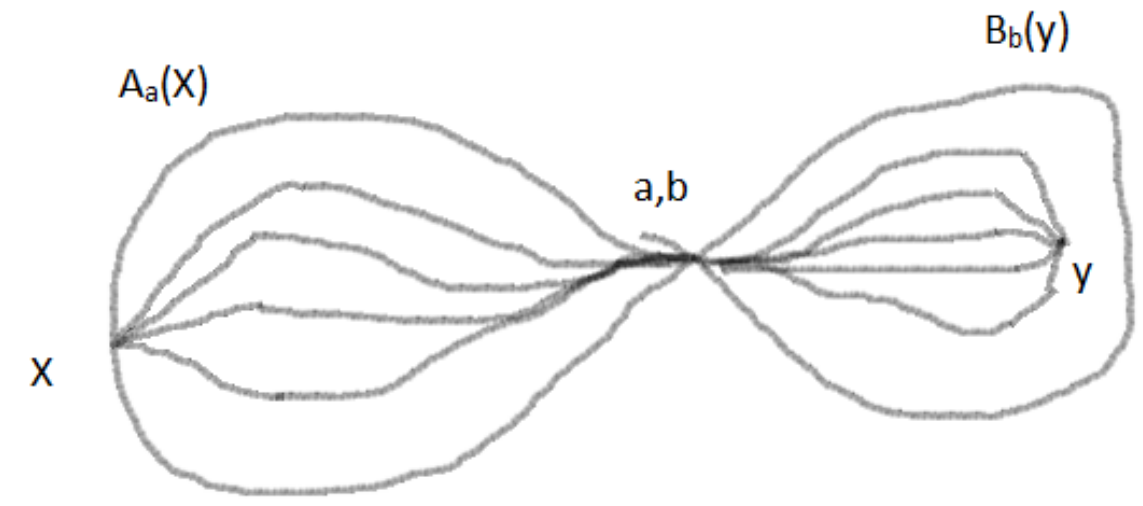

\section{weakly connected space with path}

If $x, y \in X, X=A_{x} \cup B_{y}$

Fig:3.11

A path from $x$ and $y \in \in A_{x} y \in B_{y} a, b$ are cut point of $X$ the path from $x$ to $y$ passes through either $a$ or $b$ which are cut points of $X$ 
$\Longrightarrow$ For weakly connected $X$, contains some cut points than exists path between any two non cut points $\mathrm{X}$ and $\mathrm{y}$ of $\mathrm{X}$ than each path contains at least one cut points in $\mathrm{X}$

Hence (ii) $\rightarrow$ (iii).

Lastly assume that

$\mathrm{X}$ is weekly connected and in each path in $\mathrm{X}$ contains cut points. To prove that $\mathrm{X}$ has at least two-non cut points. i.e. (iii) $\rightarrow$ (I)

Obviously, $\mathrm{X}$ is connected space. Then there exists a path between any two points in $\mathrm{X}$, because $\mathrm{X}$ contains cut points. We have for any connected space has at least two point which are non -cut points.

For $\mathrm{x}, \mathrm{y}, \in \mathrm{X}$

$\gamma(\mathrm{x} y)$ a path from $\mathrm{x}$ to $\mathrm{y}$ in $\mathrm{X}$, which are non-cut points.

\section{Lemma 3.18}

Let $\mathrm{X}$ be a path connected space. A point $\mathrm{x} \in \mathrm{X}$ be a punctured point of space. Then there exists a path from any two points on $X-\{x\}$

\section{$\underline{\text { Proof }}$}

$\overline{\text { Let } \mathrm{X}}$ be a path connected space; then there exists a path $\gamma:\left[\begin{array}{ll}0 & 1]\end{array} \rightarrow \mathrm{X}\right.$ for any point $\mathrm{y}, \mathrm{z}$ belongs to $\mathrm{X}$ and $\mathrm{x} \in \mathrm{X}$ be a punctured point of $X$

$\Longrightarrow$ There exists a path between any two points in X. As X is punctured point. Neighborhood point of $X$ are connected with $\mathrm{X}$. Each neighborhood point of $\mathrm{X}$ are connected. Then there exists path from any two point of $\mathrm{X}-\{\mathrm{x}\}$

Let $\mathrm{X}$ be a path connected space. A point $\mathrm{x} \in \mathrm{X}$ be a punctured point of a space $\mathrm{X}$ consider a path $\gamma:\left[\begin{array}{ll}0 & 1\end{array}\right] \mathrm{X}$ for any point $\mathrm{y}, \mathrm{z}$ belongs to $\mathrm{X}$ and $\mathrm{x} \in \mathrm{X}$ be a punctured point of $\mathrm{X}$. All the neighborhood points of $\mathrm{x}$ are connected with space $X-\{x\}$ say neighborhood points are $x_{i}$

$\mathrm{x}_{\mathrm{i}} \in \mathrm{X}$ as $\mathrm{x}$ does not belong to $\mathrm{X}$. As $\mathrm{X}$ is a path connected space. There exists a path $\gamma_{\mathrm{i}}$ from $\mathrm{y}$ to $\mathrm{x}_{\mathrm{i}}$ and $2^{\text {nd }}$ path $\sigma_{\mathrm{i}}$ which from $\mathrm{x}_{\mathrm{i}}$ to $\mathrm{z}$.

By definition composition

$$
\begin{aligned}
& \sigma_{i} \mathrm{O} \gamma_{\mathrm{i}}=\left\{\gamma_{\mathrm{i}}(2 \mathrm{~S}) \quad \text { if } \quad 0 \leq \mathrm{S} \leq 1 / 2\right. \\
& \text { and } \quad\left\{\sigma_{\mathrm{i}}(2 \mathrm{~S}-1) \quad \text { if } \quad 1 / 2<\mathrm{S} \leq 1\right.
\end{aligned}
$$

$\sigma o \gamma$ is path from $\mathrm{y}$ to $\mathrm{z}$ this shows that there exists a path between any two points on $\mathrm{X}-\{\mathrm{x}\}$.

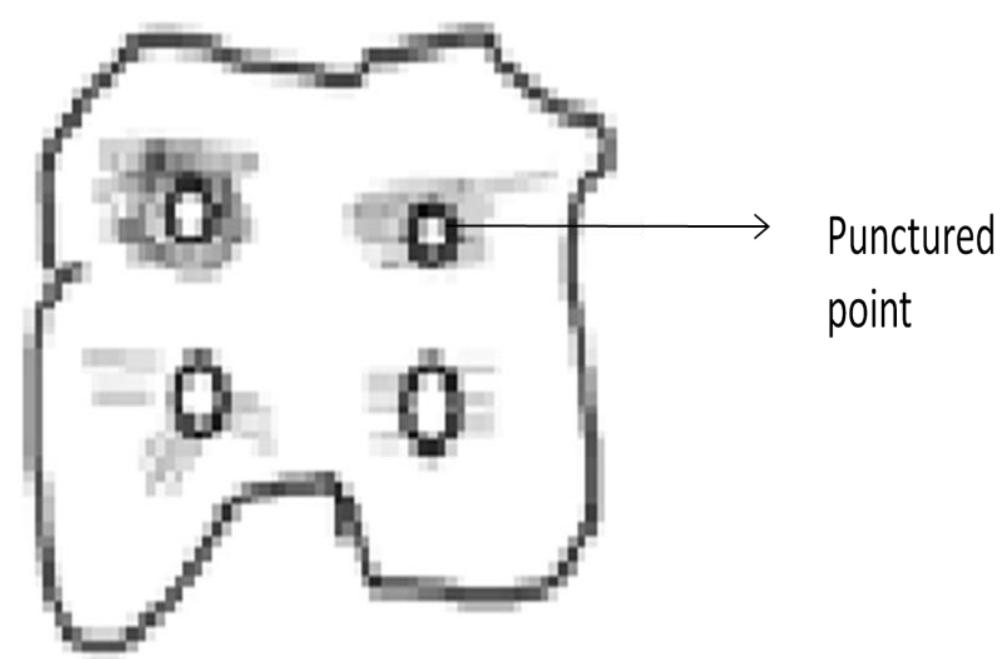

Theorem 3.19

Fig:3.12

Let $\mathrm{X}$ be a strongly connected space.

Let $\mathrm{P}(\mathrm{X})$ be a punctured point (or space) in space $\mathrm{X}$, Then 
1) There exists a path between any two points in $X$

2) The space $X$ becomes non convex Space and connected

\section{Proof}

Let $\mathrm{X}$ is strongly connected space, $\mathrm{P}(\mathrm{x})$ be a punctured point(space).

This implies $\mathrm{X}$ is path connected space even punctured point.

By theorem 3.17 and definition 2.7

There exists a path between any two points in X.

As the statement (1) if any point becomes punctured in space X, Then thereexist a path from any two point,even punctured or non- convex. Which is also continuous.

Let $\mathrm{x} \in \mathrm{X}$ be a punctured point of Xi.e. $\mathrm{X}-\{\mathrm{x}\}$ is a space without $\mathrm{x}$ i.e.hole at $\mathrm{x}$ i.e. $\mathrm{X}$ is non-convex also connected.

\section{CONCLUSION}

In this paper we modified definitions of cut points, connectedness and punctured points. These concepts, we modified and removed cut points punctured points to make connectivity stronger and stronger and applied in fiber bundles

\section{REFERENCE}

[1]. Antoni. A. Kosinski(1993)“Differential Manifolds”Academic press.iHar court Brace Jovanovich, PublisherBostonsan Diego, New York.London,Sydney, Tokyo, Toronto.

[2]. B.Honan Y. Bahrampour (1999)“Cut-points space”Proceedings of America Math Society 127(p-27972003)

[3]. Devender.K. Kamboj and Vinod Kumar(2010)“Cut point in SomeConnected Topological Spaces”. Topology and its Application 157(2010)629-634)

[4]. Devender. K. Kamboj and Vinod Kumar(2009)“H (i) Connected Topological spaces and Cutpoints"Topology and its Application 156 p(610-615)

[5]. G.T.Whyburn(1928)“Concerning the Cut point of Continue."Trans.Amer.Math.Soc.30(1928)597-609

[6]. H.G.HALOLI (2013)“Connectedness and Punctured Space in Fiber Bundle Space”International Journal of Engineering Research and Technology (IJERT) ISSN-2278-O181,Vol-2 Issue -6, June - 2013, pp-2389 to 2397.

[7]. John. M.LEE (2004)“Introduction to Topological Manifold”Springer.com(USA)

[8]. Lawrence Conlon (2001)“Differentiable Manifolds" (second edition)Birkhauser advanced textsBasler Cesrbuncher - Berlin

[9]. Marc Lackenby(2010)“Finite Covering Space of manifolds"Proceedings of the International Congress of Mathematicians. Hyderabad India(p1042-1070)

[10]. Pascal Collin,Robert Kushner,William H Meets and Harold.(2004)“The Topology Geometry and Conormal structure of properly embedded minimal surfaces" J.Differential Geometry 67(p-377-393)

[11]. Robert Bonnet and Matatyahu Rubin (2008)“A classification of continuous images of compact (co) space which are continuous images of compact ordered space.”J.Topology and its application155(2008)Elevier-Science Direct p375-411

[12]. Willam M.Boothby(2008)“An introduction to Differential Manifold and Riemannian Geometry "Academic press.

[13]. William F Basener (2006)“Topology and its applications, Wiley-Interscience”.A John Wiley \& sons Inc.Publication 